\title{
Review of Approaches to Developing Intersegmental Plane during Segmentectomy
}

\author{
Xingshi Chen ${ }^{1, *}$ Zhengyuan Zhang ${ }^{1, *}$ Ning $\mathrm{Xu}^{2, *}$ Dongchun $\mathrm{Ma}^{2}$ Hecheng $\mathrm{Li}^{1}$ \\ ${ }^{1}$ Department of Thoracic Surgery, Ruijin Hospital, Shanghai Jiao Tong \\ University School of Medicine, Shanghai, China \\ 2 Department of Thoracic Surgery, Anhui Chest Hospital, Hefei, Anhui \\ Province, China

\begin{abstract}
Address for correspondence Hecheng Li, MD, PhD, Department of Thoracic Surgery, Ruijin Hospital, Shanghai Jiao Tong University School of Medicine, No. 197, Rui Jin Er Road, Shanghai 200025, China (e-mail: lihecheng2000@hotmail.com).
\end{abstract}

Thorac Cardiovasc Surg 2022;70:341-345.
Abstract
Keywords
- lung cancer treatment
- surgery
- medical
- surgical equipment
- instruments
- sutures
- outcomes
- mortality
- morbidity
- tumor

In recent years, with the popularity of computed tomography (CT) scanning, early lung cancer has been found in a large number of patients, and segmentectomy has been widely used in clinical practice. The development of intersegmental plane is the most critical step in segmentectomy. At present, there are many methods to identify the intersegmental plane. Also, dissection of the intersegmental plane has been a challenge for thoracic surgeons for decades because of the complicated anatomic variations. This study focuses on the safety and efficacy of relevant methods in both identification and dissection of the intersegmental plane in segmentectomy.

\section{Introduction}

With the popularization of low-dose computed tomography (LDCT) applied to lung cancer screening, more and more patients with ground glass opacity (GGO) have been detected. ${ }^{1,2}$ Segmentectomy is accepted as one of the standard surgical procedures for small pulmonary nodules and GGOs, ${ }^{3}$ as its safety and oncological outcomes have been proved by several studies. ${ }^{4-8}$ It is well known that identification of the intersegmental plane is a key step in segmentectomy. Inaccurate recognition of the intersegmental plane may lead to dysfunction of the remaining lung tissue, mismatching of ventilation or blood flow, or long-term air leakage after surgery,

All three authors contributed equally to the article.

received

December 13, 2020

accepted after revision

January 25, 2021

published online

February 16, 2021 which even requires unplanned secondary surgery. ${ }^{9}$ On the other hand, dissection of the intersegmental plane in segmentectomy is also challenging for thoracic surgeons because of the complicated anatomy and anatomical variations, along with the unclear boundary between pulmonary segments. This review will discuss the present state of methods for developing the intersegmental plane, focusing on their current application and comparison.

\section{Identification of Intersegmental Plane}

In our opinion, an ideal method of identifying the intersegmental plane should have the following characteristics: accurate, efficient, convenient, and repeatable. Theoretically, the intersegmental plane can be defined from lung surface and lung parenchyma (intersegmental veins). Many methods

\footnotetext{
(C) 2021. The Author(s).

This is an open access article published by Thieme under the terms of the Creative Commons Attribution-NonDerivative-NonCommercial-License, permitting copying and reproduction so long as the original work is given appropriate credit. Contents may not be used for commercial purposes, or adapted, remixed, transformed or built upon. (https://creativecommons.org/ licenses/by-nc-nd/4.0/) Georg Thieme Verlag KG, Rüdigerstraße 14, 70469 Stuttgart, Germany
} 
have been applied to indicate the intersegmental boundary on lung surface, of which modified inflation-deflation, selective segmental high-frequency ventilation, and indocyanine green (ICG) fluorescence are the most widely used methods. Also, intersegmental veins are usually distinguished to verify the accuracy of other methods.

\section{Modified Inflation-Deflation Method}

After double lumen tube intubation, in a state of unilateral differential ventilation, the targeted segment bronchus, artery, and vein (except for intersegmental veins) are identified and dissected by ligation or stapler device. Then the collapsed lung is re-expanded completely with controlled airway pressure under $\sim 20 \mathrm{~cm} \mathrm{H} \mathrm{H}_{2} \mathrm{O}$ using pure $\mathrm{O}_{2}$. In this condition, the bronchus-dissected segment can also be inflated by the airstream passing through the pores of Kohn. Then, the affected side will be open to atmosphere while continuing ventilation of the contralateral lung tissue. Ten to 20 minutes later, an irregular boundary line will occur gradually between the inflated targeted segment and the deflated preserved segments, which represent the intersegmental border. ${ }^{10}$

Besides its safety and accuracy, this method does not require additional equipment. The boundary line can occur for a relatively long time and it is suitable for all segments. Nevertheless, there are disadvantages such as dependence on 3D-CT, long waiting period, and interfering the vision of the surgical field. In addition, this method is not applicable for patients with emphysema and pleural adhesions. ${ }^{11}$

\section{Selective Segmental High-Frequency Ventilation} In 2003 and 2007, Matsuoka et $\mathrm{al}^{12}$ and Okada et al, ${ }^{13}$ respectively, proposed this technique to develop the intersegmental plane. After isolation of the bronchus of the targeted segment, the anesthesiologist puts a bronchofiberscopy through the double-lumen tube into the orifice of the targeted segmental bronchus, so that the surgeon can see the light of the bronchoscope and lead it to the suitable position of the targeted bronchus with high-frequency jet ventilation $(40 \mathrm{~Hz}$, working pressure of $2 \mathrm{~kg} / \mathrm{cm}^{2}$ ) to inflate the segment. Then, the target segment's bronchial cord is ligated to inflate the target segment continually while the preserved segments are deflated, which will produce an inflation-deflation line.

This method can identify the intersegmental plane quickly, and the selective target segment ventilation affects little on the visual field of thoracoscopy. On the other hand, the location of the target segment bronchus is complicated, which also requires the cooperation of an experienced anesthesiologist. Moreover, the diameter of bronchofiberscopy should be adjusted according to the different diameter of segmental bronchus or subsegmental bronchus. These adverse factors limit its accessibility.

\section{Indocyanine Green Fluorescence}

In 2009, Misaki et al ${ }^{14}$ first reported the application of ICG in the recognition of intersegmental plane. After the corresponding pulmonary artery of the target segment has been ligated, ICG is administered intravenously during infrared thoracoscopy (IRT). This is called negative staining. The IRT emits 805 and $940 \mathrm{~nm}$ infrared light, and the preserved lung tissue containing ICG absorbs 805-nm light, reflecting 940-nm light, which turns blue under IRT. Meanwhile, the target segment does not contain ICG and shows white, identifying the intersegmental plane.

This method is fast and accurate, and can be applied to patients with emphysema as well as avoiding the interference in the visual field caused by the expanded lung. However, there are disadvantages such as the risk of ICG allergy, the need for additional equipment, and the short duration of dyeing. Specifically, some studies have shown that ICG cannot be used in patients with poor liver function, ICG allergy, or iodine allergy. ${ }^{11}$ And ICG exists in the pulmonary circulation for a short time and just marks the boundary on the surface. When it comes to complex segment resection that requires a large amount or repeated use of ICG, multiple use of ICG may lead the ICG to enter the target lung tissue through the bronchial circulation, and also increase the risk. What's more, IRT or Da Vinci Si/Xi system costs a lot.

Another way is positive staining. This method identifies intersegmental plane of the lung with infrared fluorescence imaging after transbronchial injection of ICG, ${ }^{15,16}$ which is more convenient for joint or subsegmentectomy. The disadvantage is the difficulty of the identification of bronchus, and there is possibility of contrast agent reflux causing confusion.

In addition, the study of Mehta et $\mathrm{al}^{17}$ indicated that the application of ICG may allow for extended oncological margins on the resected specimen in segmentectomy.

\section{Dissection of the Intersegmental Plane}

The main objective of this step is to obtain sufficient margin, ${ }^{18,19}$ preserve lung function, and reduce complication incidence. Currently, there are two main approaches to dissecting intersegmental planes: using stapling devices ${ }^{20-22}$ or energy instruments. ${ }^{13,23-25}$

\section{Stapling Devices}

This is the most common way to dissect the intersegmental plane in clinical practice. After properly dividing the relevant vessels and bronchus, and recognizing the intersegmental plane, the target segment would be dissected along the determined boundary with stapling devices.

In 2019, Ojanguren et $\mathrm{al}^{20}$ analyzed 175 patients who received dissection of the intersegmental plane by stapling devices. The overall postoperative complication rate was $17 \%$, the serious complication rate was $0.6 \%$, the average length of postoperative chest drainage was 3 days, and the average length of hospital stay was 5.7 days. The chest radiography at the time of discharge and 1 month after surgery indicated that the incidence of incomplete lung re-expansion was 7.4 and $2.8 \%$, respectively. Among them, patients who underwent upper lobe segmentectomy had significantly more incomplete re-expansion. Therefore, the author believes that the stapling device has little effect on lung re-expansion. Endoh et $\mathrm{al}^{21}$ reported 20 cases of dorsal (S10) and lateral dorsal segments $(S 9+10)$, all of which used stapling devices to dissect the intersegmental plane. Only one patient among them had atelectasis. Hence, judging from the results above, the 
incidence of postoperative atelectasis or incomplete lung re-expansion will not increase when stapling device is used to dissect the intersegmental plane.

There are also reports on the perioperative safety of using stapling devices to divide intersegmental plane. In 2012, Pardolesi et $\mathrm{al}^{22}$ reported 17 cases of robot-assisted lung resection, all of which used stapling devices to dissect the intersegmental plane. The average operation time was 189 minutes, and no serious complications occurred during the operation. The postoperative complications rate was $17.6 \%$, including one case of pneumonia and two cases of air leakage. The length of median hospital stay after surgery was 5 days. Zhao et $\mathrm{al}^{26}$ also used stapling devices to deal with the intersegmental plane. In their study of comparing the safety and efficacy between segmentectomy and lobectomy, the results showed that in the perioperative outcomes such as intraoperative blood loss, operation time, chest drainage time, length of hospital stay, postoperative complications, etc., segmentectomy with stapling devices to separate the intersegmental plane had no difference with lobectomy.

\section{Energy Instruments}

The energy instruments used in segmentectomy to separate the intersegmental plane include electrocautery, ultrasonic scalpel, LigaSure, etc. Due to the flexibility of the energy instruments, the area of resection is determined by the surgeon to a greater degree, which ensures the reliability of the resection margin, especially in the treatment of tumors close to the intersegmental plane. Several studies have confirmed its feasibility and safety.

In 2009, Oizumi et $\mathrm{al}^{23}$ reported 28 cases of total thoracoscopic pulmonary segmentectomy, in which electrocautery was used to separate the intersegmental plane. The median operation time was 216 minutes, and the duration chest drainage was 1 to 7 days (median duration: 1 day). One patient developed subcutaneous emphysema that spontaneously recovered. No mortality was observed for 30 days after the surgery. Okada et $\mathrm{al}^{13}$ used selective segmental high-frequency ventilation to determine the intersegmental plane, and then separated it with electrocautery. A total of 52 consecutive cases were included in the study. The median operation time was 155 minutes, and the median blood loss volume was $60 \mathrm{~mL}$. The postoperative complication rate was $13.5 \%$. The most common complication was air leakage, with a median air leakage time of 1 day. Thus, these two studies demonstrated the feasibility and safety of the electrocautery to dissect the intersegmental plane.

In 2010, Takagi et $\mathrm{al}^{24}$ reported 28 cases of segmentectomy using energy instruments to dissect the intersegmental plane. Seventeen cases used electrocautery and 11 cases used ultrasonic scalpel. There were no serious complications after the operation, but eight cases (three cases in electrocautery group and five cases in ultrasonic scalpel group) developed pulmonary fistula within 1 to 3 months after surgery, and the histological findings of the cutting surface showed that most of the layer of coagulation necrosis by the ultrasonic scalpel was 2-mm thick and it was denser than that of electrocautery. Therefore, the author believed that the small bronchial stump could not tolerate the airway pressure because the thick coagulation necrosis delayed healing of the postoperative wound. It was necessary to ligate the stump of small bronchus, even though the stump had been temporally closed by coagulation necrosis with the electrocautery or ultrasonic scalpel during the operation.

LigaSure has been widely used clinically in recent years. In 2016, Kuroda et $\mathrm{al}^{25}$ compared 12 patients who accepted the LigaSure technique for dissection of intersegmental plane during thoracoscopic anatomical segmentectomy with 38 patients who used the stapling devices. The mean durations for dissection of the intersegmental plane in the two groups were 22.8 and 16.2 minutes. In the LigaSure group, 3 cases had early mild air leakage, and 1 case had delayed mild air leakage, whereas in the stapling device group, 1 case had postoperative prolonged air leakage, 1 case had early mild air leakage, and 1 case had delayed mild air leakage. The authors believed that LigaSure has no disadvantages compared with stapling devices, but considering the difference in the sample size, the LigaSure group might have a tendency to increase the incidence of postoperative air leakage.

\section{Comparison between Stapling Devices and Energy Instruments}

Due to its relatively weak closure effect on the residual surface, it is theoretically speculated that the energy instrument may increase the incidence of postoperative air leakage and other complications, but it can reduce the cost of medical materials. However, there are few prospective studies comparing the advantages and disadvantages of these two methods. In 2020, our center reported a randomized controlled trial to compare the perioperative outcomes of dissecting the intersegmental plane by stapling devices and energy instruments. ${ }^{27}$ The study showed the incidence of postoperative complications (e.g., air leakage) was higher in the electrocautery group than in the stapler device group (11/32 [34.4\%] vs. $2 / 33$ [6.1\%], $p=0.004)$. There were no differences in duration of operation, blood loss during operation, first-day drainage volume, duration of drainage, postoperative hospital stays, loss of lung function, or total medical cost, although the per patient cost of medical materials was higher in the stapler device group (US\$ $4214.6 \pm 1185.4$ vs. US\$ $3260.1 \pm 852.6, p<0.001)$. Therefore, we believed that among patients undergoing segmentectomy, the use of stapling devices to divide intersegmental planes decreased postoperative complications without further compromising lung function or increasing economic burden.

Other studies focusing on this point are mostly retrospective design, and the sample sizes are limited, so no clear conclusion has been reached. In 2011, Miyasaka et $\mathrm{al}^{28}$ reviewed 49 cases of segmentectomy; 18 cases used stapling devices and 31 cases used electrocautery to separate the intersegmental plane. The study did not find significant differences in perioperative outcomes between the two groups. Multivariate analysis showed that postoperative complications were only related to the position of target segment and the intraoperative blood loss volume but had nothing to do with the method of dissecting the intersegmental plane. Ohtsuka et al $^{29}$ reviewed 47 cases of segmentectomy, of which 22 cases used an electrocautery to separate the intersegmental 
plane and 25 cases used electrocautery combined with stapling devices. The study showed that there was no statistical difference between electrocautery alone and in combination with stapling devices in the duration of surgery, intraoperative blood loss, duration of chest drainage, postoperative hospital stays, postoperative loss of FEV1, loss of FVC, or incidence of postoperative complications. However, incidence of prolonged air leak was higher in the electrocautery-alone group (14\% [3/22] vs. $4 \%$ [1/25], $p=0.025)]$. Similarly, Tao et al $^{30}$ also reported that stapling devices did not lead to less preserved volume or function than electrocautery in the dissection of the intersegmental plane.

\section{Conclusion}

Pulmonary anatomical segmentectomy is now accepted by more and more medical centers and thoracic surgeons. As the key step of segmentectomy, the development of intersegmental plane is attracting growing attention. In our opinion, all the above-mentioned methods for identifying the intersegmental plane have their advantages and disadvantages. The choice of the method mainly depends on the surgeon's preference, clinical characteristics of the patient, and other objective conditions. On the other hand, according to our prospective study, combined with the findings of several existing retrospective studies, we recommend the use of stapling devices as a better method for dissection of the intersegmental plane.

\section{Funding}

This study received funding from the National Natural Science Foundation of China (8187102600, 82072557), Outstanding Academic Leader of Shanghai (20XD1402300), Ruijin Youth NSFC Cultivation Fund (2019QNPY01052), and the Shanghai Municipal Education Commission-Gaofeng Clinical Medicine Grant Support (20172005).

\section{Conflict of Interest \\ None declared.}

\section{Acknowledgments}

All the authors contributed to the study design, literature search, and writing. D.M. and H.L. were the study supervisors. Dr. Xingshi Chen, Dr. Zhengyuan Zhang, and Dr. Ning Xu contributed to the work equally, and Profs. Hecheng Li and Dongchun Ma are both corresponding authors.

\section{Reference}

1 Kidane B, Yasufuku K. Advances in image-guided thoracic surgery. Thorac Surg Clin 2016;26(02):129-138

2 Yang W, Qian F, Teng J, et al; Written on behalf of the AME Thoracic Surgery Collaborative Group. Community-based lung cancer screening with low-dose CT in China: results of the baseline screening. Lung Cancer 2018;117:20-26

3 National Comprehensive Cancer Network. Non-Small Cell Lung Cancer (Version 3. 2018https://www.nccn.org/professionals/ physician_gls/pdf/nscl.pdf. Accessed February 21, 2018
4 Hwang Y, Kang CH, Kim HS, Jeon JH, Park IK, Kim YT. Comparison of thoracoscopic segmentectomy and thoracoscopic lobectomy on the patients with non-small cell lung cancer: a propensity score matching study. Eur J Cardiothorac Surg 2015;48(02): 273-278

5 Kodama K, Higashiyama M, Okami J, et al. Oncologic outcomes of segmentectomy versus lobectomy for clinical T1a NOM0 nonsmall cell lung cancer. Ann Thorac Surg 2016;101(02):504-511

6 Landreneau RJ, Normolle DP, Christie NA, et al. Recurrence and survival outcomes after anatomic segmentectomy versus lobectomy for clinical stage I non-small-cell lung cancer: a propensitymatched analysis. J Clin Oncol 2014;32(23):2449-2455

7 Okada M, Mimae T, Tsutani Y, et al. Segmentectomy versus lobectomy for clinical stage IA lung adenocarcinoma. Ann Cardiothorac Surg 2014;3(02):153-159

8 Tsubokawa N, Tsutani Y, Miyata Y, et al. Segmentectomy versus lobectomy for radiologically pure solid clinical T1a-bNOM0 lung cancer. World J Surg 2018;42(08):2493-2501

9 Nakazawa S, Shimizu K, Mogi A, Kuwano H. VATS segmentectomy: past, present, and future. Gen Thorac Cardiovasc Surg 2018;66 (02):81-90

10 Wang J, Xu X, Wen W, Wu W, Zhu Q, Chen L. Modified method for distinguishing the intersegmental border for lung segmentectomy. Thorac Cancer 2018;9(02):330-333

11 Jin Y, Wang M, Xue L, Zhao X. Clinical application of near-infrared thoracoscopy with indocyanine green in video-assisted thoracoscopic anatomical segmentectomy. Surg Innov 2019;26(04): 473-477

12 Matsuoka H, Nishio W, Sakamoto T, Harada H, Yoshimura M, Tsubota N. Selective segmental jet injection to distinguish the intersegmental plane using jet ventilation. Jpn J Thorac Cardiovasc Surg 2003;51(08):400-401

13 Okada M, Mimura T, Ikegaki J, Katoh H, Itoh H, Tsubota N. A novel video-assisted anatomic segmentectomy technique: selective segmental inflation via bronchofiberoptic jet followed by cautery cutting. J Thorac Cardiovasc Surg 2007;133(03):753-758

14 Misaki N, Chang SS, Gotoh M, Yamamoto Y, Satoh K, Yokomise H. A novel method for determining adjacent lung segments with infrared thoracoscopy. J Thorac Cardiovasc Surg 2009;138(03): 613-618

15 Sekine Y, Ko E, Oishi H, Miwa M. A simple and effective technique for identification of intersegmental planes by infrared thoracoscopy after transbronchial injection of indocyanine green. J Thorac Cardiovasc Surg 2012;143(06):1330-1335

16 Oh S, Suzuki K, Miyasaka Y, Matsunaga T, Tsushima Y, Takamochi $\mathrm{K}$. New technique for lung segmentectomy using indocyanine green injection. Ann Thorac Surg 2013;95(06):2188-2190

17 Mehta M, Patel YS, Yasufuku K, et al. Near-infrared mapping with indocyanine green is associated with an increase in oncological margin length in minimally invasive segmentectomy. J Thorac Cardiovasc Surg 2019;157(05):2029-2035

18 Ginsberg RJ, Rubinstein LVLung Cancer Study Group. Randomized trial of lobectomy versus limited resection for T1 N0 non-small cell lung cancer. Ann Thorac Surg 1995;60(03):615-622, discussion 622-623

19 Howington JA, Blum MG, Chang AC, Balekian AA, Murthy SC. Treatment of stage I and II non-small cell lung cancer: diagnosis and management of lung cancer, 3rd ed: American College of Chest Physicians evidence-based clinical practice guidelines. Chest 2013;143(5, Suppl):e278S-e313S

20 Ojanguren A, Gossot D, Seguin-Givelet A. Division of the intersegmental plane during thoracoscopic segmentectomy: is stapling an issue? J Thorac Dis 2016;8(08):2158-2164

21 Endoh M, Oizumi H, Kato H, et al. Posterior approach to thoracoscopic pulmonary segmentectomy of the dorsal basal segment: a single-institute retrospective review. J Thorac Cardiovasc Surg 2017;154(04):1432-1439 
22 Pardolesi A, Park B, Petrella F, Borri A, Gasparri R, Veronesi G. Robotic anatomic segmentectomy of the lung: technical aspects and initial results. Ann Thorac Surg 2012;94(03):929-934

23 Oizumi H, Kanauchi N, Kato H, et al. Total thoracoscopic pulmonary segmentectomy. Eur J Cardiothorac Surg 2009;36(02):374-377 , discussion 377

24 Takagi K, Hata Y, Sasamoto S, et al. Late onset postoperative pulmonary fistula following a pulmonary segmentectomy using electrocautery or a harmonic scalpel. Ann Thorac Cardiovasc Surg 2010;16(01):21-25

25 Kuroda H, Dejima H, Mizumo T, Sakakura N, Sakao Y. A new LigaSure technique for the formation of segmental plane by intravenous indocyanine green fluorescence during thoracoscopic anatomical segmentectomy. J Thorac Dis 2016;8(06): 1210-1216

26 Zhao X, Qian L, Luo Q Huang J. Segmentectomy as a safe and equally effective surgical option under complete video-assisted thoracic surgery for patients of stage I non-small cell lung cancer. J Cardiothorac Surg 2013;8:116

27 Chen X, Jin R, Xiang J, et al. Methods for dissecting intersegmental planes in segmentectomy: a randomized controlled trial. Ann Thorac Surg 2020;110(01):258-264

28 Miyasaka Y, Oh S, Takahashi N, Takamochi K, Suzuki K. Postoperative complications and respiratory function following segmentectomy of the lung: comparison of the methods of making an inter-segmental plane. Interact Cardiovasc Thorac Surg 2011;12 (03):426-429

29 Ohtsuka T, Goto T, Anraku M, et al. Dissection of lung parenchyma using electrocautery is a safe and acceptable method for anatomical sublobar resection. J Cardiothorac Surg 2012;7:42

30 Tao H, Tanaka T, Hayashi T, et al. Influence of stapling the intersegmental planes on lung volume and function after segmentectomy. Interact Cardiovasc Thorac Surg 2016;23(04): $548-552$ 